\title{
Standardization of Seed Biopriming with Liquid Biofertilizers on Snake Gourd (Trichosanthes cucumerina)
}

\author{
U. Gowthamy ${ }^{1 *}$, P. Selvaraju ${ }^{2}$ and G. Hemalatha ${ }^{1}$ \\ ${ }^{1}$ Department of Seed Science and Technology, Tamil Nadu Agricultural University, \\ Coimbatore-641 003, Tamil Nadu, India \\ ${ }^{2}$ Seed Centre, Tamil Nadu Agricultural University, Coimbatore-641 003, Tamil Nadu, India \\ *Corresponding author
}

\section{A B S T R A C T}

\begin{abstract}
Keywords
Snakegourd

(Trichosanthes

cucumerina),

Azotobacter,

Azospirillum and

phosphobacteria,

Seed biopriming.

Article Info

Accepted:

19 October 2017

Available Online:

10 December 2017

Biopriming is a very important seed invigoration technique for rapid germination. In order to utilize the biopriming influence on seedling growth in snake gourd (Trichosanthes cucumerina), this experiment was conducted to study the seed biopriming with liquid biofertilizers on snakegourd cv. $\mathrm{CO} 2$ for better germination and seedling growth. The results showed that the effect of biopriming was significant on germination percentage, seedling dry weight and seedling vigour in snakegourd. Seeds bioprimed with Azospirillum $20 \%$ concentration for $18 \mathrm{~h}$ was the best and suitable biopriming treatment. This treatment showed a higher speed of germination (5.6), percentage of germination (84\%), dry matter production (1.187g 10 seedlings $^{1}$ ) and vigour index (3969) compared to other concentrations, durations and over the other treatments.
\end{abstract}

\section{Introduction}

Snake gourd (Trichosanthes cucumerina) also known as Chinese cucumber belongs to the family Cucurbitaceae. It is called potlakaaya in Telugu, pudalankaai in Tamil, dhundul in Assamese, paduvalakaayi in Kannada and padavalanga in Malayalam. It occupies an important place among vegetables in India. It is an annual plant with rapid growth and of climbing habit. The fruits are large and greenish white. They often reach upto 150 $\mathrm{cm}$. in length and $8 \mathrm{~cm}$. in thickness. There is also a short-fruited type. Tender fruits are used as vegetables. Snake gourd is a very nutritious vegetable. An analysis of this vegetable shows it consists of moisture 94.6 percent, protein 0.5 percent, fat 0.3 percent, fibre 0.8 percent and carbohydrate 3.3 percent per 100 grams of edible portion. Snakegourd is cultivated in Tamil Nadu in larger area with an average productivity of 18 tonnes ha- ${ }^{-1}$.

For any crop production, seed is the basic input and if the seed is not having good germination, the optimum population in the field can't be maintained which ultimately affect the crop yield. In snakegourd, normally the germination is below 60 per cent and by any presowing treatment if the germination is 
improved it would help in maintaining the required population in the field. Biopriming is one of the presowing treatments that can improve the germination and vigour of the seedling.

Research on biopriming of snakegourd seeds using liquid biofertilizers, particularly their effects on germination and seedling vigour is lacking. Hence the present study was designed to investigate the beneficial effects of biopriming on snake gourd using liquid biofertlizers.

\section{Materials and Methods}

Genetically pure seeds of snakegourd cv.CO2, a short duration variety obtained from Susi seeds international, Mylapore, Chennai of Tamil Nadu, formed the base material for this study. The laboratory studies were carried out at the Department of Seed Science and Technology, Tamil Nadu Agricultural University, Coimbatore. Five treatments viz., non-primed seed (control), hydropriming, biopriming with Azotobactor, Azospirillum and phosphobacteria were taken up in this study. The experiment was carried out with four replications in Completely Randomized Block Design (CRD). In order to standardize the optimum concentration of liquid biofertilizers and duration. Liquid biofertilizers were prepared at 10, 15 and 20 percent concentration. Seeds were soaked in equal volume of solution in different concentrations in each of the biopriming agents. For hydropriming, simple water is used for soaking. The nonprimed seeds formed the control. After soaking, the seeds were removed from the solutions and shade dried at room temperature for assessing the seed quality parameters. The number of seeds germinated on each day was recorded daily upto $14^{\text {th }}$ day. After $14^{\text {th }}$ day of germination, their performance was evaluated for germination (\%), dry matter production ( $\mathrm{g}$ seedlings ${ }^{-10}$ ) and vigour index. Then best treatments one each from, Azotobactor, Azospirillum, phosphobacteria, hydroprimed seed and control were standardized.

Liquid biofertilizers were diluted to 10,15 and $20 \%$ concentrations.

\section{Speed of germination}

Four replicates of twenty five seeds each were used to test the speed of germination of seeds in sand medium. The seeds showing radicle protrusion were counted daily from third day after sowing until fourteenth day. From the number of seeds germinated on each day, the speed of germination was calculated using the following formula and the results were expressed in number (Maguire, 1962).

Speed of germination $=\frac{X_{1}}{Y_{1}}+\frac{X_{2}-X_{1}}{Y_{2}}+\ldots \ldots \ldots . .+\frac{X_{n}-X_{n-1}}{Y_{n}}$

$\mathrm{X}_{1}$ - Number of seeds germinated at first count

$\mathrm{X}_{2^{-}}$Number of seeds germinated at second count

$X_{n}$ - Number of seeds germinated on $n^{\text {th }}$ day

$Y_{1-}$ Number of days from sowing to first count

$\mathrm{Y}_{2^{-}}$Number of days from sowing to second count

$\mathrm{Y}_{\mathrm{n}}$ - Number of days from sowing to $\mathrm{n}^{\text {th }}$ count

\section{Germination}

The seeds were sown in sand medium prepared as per ISTA procedures (2009) and the seeds were sown in four replications of 100 seeds each and were placed in a germination room maintained at $25 \pm 2{ }^{\circ} \mathrm{C}$ temperature and $90 \pm 3 \%$ relative humidity. After the germination period of 14 days, the seedlings were evaluated as normal and 
abnormal seedlings and dead seeds. Based on the normal seedlings, the germination percentage was calculated adopting the following formula.

Number of seeds germinated Seed germination $(\%)=$ X 100

Total number of seeds sown

\section{Root length (cm)}

At the time of germination count, ten normal seedlings were selected at random from each replication and used for measuring the root length of seedlings. Root length was measured from the point of attachment of seed to the tip of primary root. The mean values were calculated and expressed in centimetre.

\section{Shoot length $(\mathrm{cm})$}

The seedlings used for measuring root length were also used for measuring shoot length. The shoot length was measured from the point of attachment of seed to tip of the leaf and the mean values were expressed in centimetre.

\section{Dry matter production 10 seedlings $^{-1}(\mathrm{~g})$}

Ten normal seedlings were placed in a paper cover and dried in shade for $24 \mathrm{~h}$ and then, they were kept in an oven maintained at $80 \pm$ $2^{\circ} \mathrm{C}$ for $24 \pm 1 \mathrm{~h}$. The dried seedlings were weighed and the mean values were expressed in $\mathrm{mg} 10$ seedlings ${ }^{-1}$.

\section{Vigour index}

Vigour index values were computed using the following formula and the mean values were expressed in whole number (Abdul-Baki and Anderson, 1973).

Vigour index $=$ Germination $(\%) \mathrm{x}$ Seedling length $(\mathrm{cm})$.

\section{Statistical analysis}

The data obtained from different treatments were analysed for the ' $F$ ' test of significance following the methods described by Panse and Sukatme (1985). Wherever necessary, the per cent values were transformed to angular (Arc-sine) values before analysis. The critical differences $(C D)$ were calculated at 5 per cent probability level. The data were tested for statistical significance. If the $\mathrm{F}$ test is nonsignificant, it was indicated by the letters NS.

\section{Results and Discussion}

Significant variations were observed in speed of germination, germination, drymatter production, seedling length and vigour index by biopriming treatments, durations of biopriming and their interactions (Tables 118).

The interactions effects indicated the best performance of seeds bioprimed with Azotobacter $10 \%$ for $30 \mathrm{~h}$ (6.2). The lowest speed of germination of 3.2 was noticed in nonprimed seeds.

The interactions, seeds bioprimed with Azotobacter at $20 \%$ for $30 \mathrm{~h}$ recorded higher germination ( 80 per cent) than nonprimed seeds.

The interactions between the biopriming treatments and durations revealed that Azotobacter $20 \%$ biopriming for $30 \mathrm{~h}$ measured the longest root of $19.5 \mathrm{~cm}$. The shortest root was observed in nonprimed seeds $(14.8 \mathrm{~cm})$.

The interactions effects showed that Azotobacter $20 \%$ for $30 \mathrm{~h}$ produced the longest shoot of $29.7 \mathrm{~cm}$ followed by Azotobacter $15 \%$ for $30 \mathrm{~h}(29.1 \mathrm{~cm})$. The shortest root of $23.4 \mathrm{~cm}$ was produced in nonprimed seeds. 
From the interactions, it was observed that seeds bioprimed with $20 \%$ Azotobacter for $30 \mathrm{~h}$ produced higher drymatter production (1.186g 10 seedlings $^{-1}$ ) followed by Azotobacter $15 \%$ for $30 \mathrm{~h} \quad(1.181 \mathrm{~g} \quad 10$ seedlings $\left.{ }^{-1}\right)$. The drymatter production was

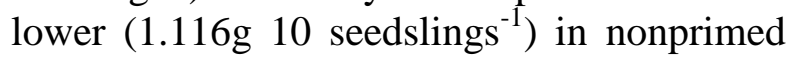
seeds. Regarding the interactions effects, the Azotobacter $20 \%$ for $30 \mathrm{~h}$ registered better vigour index (3847) than the nonprimed seeds (2334).

Significant variations was observed in Speed of germination, Germination, Drymatter production, seedling length and vigour index by biopriming treatments, durations of biopriming and their interactions.The interactions effects indicated the better performance of seeds hydroprimed for $18 \mathrm{~h}$ (6.2). The lowest speed of germination of 3.2 was noticed in nonprimed seeds.

Regarding the interactions, seeds bioprimed with Azospirillum $20 \%$ for $18 \mathrm{~h}$ recorded higher germination (84 per cent) than nonprimed seeds (61 per cent). The interactions between the biopriming treatments and durations revealed that Azospirillum 20\% biopriming for $18 \mathrm{~h}$ measured the longest root of $20.0 \mathrm{~cm}$. The shortest root was observed in nonprimed seeds $(14.9 \mathrm{~cm})$.

The interactions effects showed that Azospirillum $20 \%$ for $18 \mathrm{~h}$ produced the longest shoot of $30.2 \mathrm{~cm}$ and the shortest root was with nonprimed seeds $(23.8 \mathrm{~cm})$.

From the interactions, it was observed that seeds bioprimed with $20 \%$ Azospirillum for $18 \mathrm{~h}$ produced higher drymatter production $\left(1.187 \mathrm{~g} \quad 10 \quad\right.$ seedlings $\left.^{-1}\right)$ followed by Azospirillum $15 \%$ for $18 \mathrm{~h} \quad(1.182 \mathrm{~g} \quad 10$ seedlings $^{-1}$ ).

The drymatter production was lower $(1.117 \mathrm{~g}$ 10 seedlings $^{-1}$ ) in nonprimed seeds.

Regarding the interactions effects, the Azospirillum biopriming at $20 \%$ for $18 \mathrm{~h}$ registered better vigour index (3969) than nonprimed seeds (2368).

The details on biopriming agents, their concentrations and durations of soaking are given in the table below

\begin{tabular}{|c|c|c|}
\hline Biopriming agents & Concentrations (\%) & Durations of soaking (h) \\
\hline Nonprimed seed & - & - \\
\hline Water & - & 12,1824 and 30 \\
\hline \multirow{3}{*}{ Azotobacter } & 10 & \multirow{3}{*}{$12,18,24$ and 30} \\
\hline & 15 & \\
\hline & 20 & \\
\hline \multirow{3}{*}{ Azospirillum } & 10 & \multirow{3}{*}{$12,18,24$ and 30} \\
\hline & 15 & \\
\hline & 20 & \\
\hline \multirow{3}{*}{ Phosphobacteria } & 10 & \multirow{3}{*}{$12,18,24$ and 30} \\
\hline & 15 & \\
\hline & 20 & \\
\hline
\end{tabular}


Table.1 Standardization of seed biopriming using Azotobacter for snakegourd cv. $\mathrm{CO}_{2}-$ Speed of germination

\begin{tabular}{|c|c|c|c|c|c|c|}
\hline \multirow{3}{*}{\multicolumn{2}{|c|}{$\begin{array}{c}\text { Biopriming treatments } \\
\text { (T) }\end{array}$}} & \multicolumn{5}{|c|}{ Speed of germination } \\
\hline & & \multicolumn{5}{|c|}{ Soaking duration in $h(D)$} \\
\hline & & 12 & 18 & 24 & 30 & Mean \\
\hline \multirow{5}{*}{$\begin{array}{l}\text { Nonprimed s } \\
\text { Hydroprimin } \\
\text { Azotobacter }\end{array}$} & & 3.2 & 3.2 & 3.2 & 3.2 & 3.2 \\
\hline & & 4.3 & 4.3 & 4.9 & 4.2 & 4.4 \\
\hline & $10 \%$ & 4.3 & 4.3 & 4.4 & 6.2 & 4.8 \\
\hline & $15 \%$ & 4.8 & 4.4 & 4.7 & 4.6 & 4.6 \\
\hline & $20 \%$ & 5.0 & 4.5 & 5.0 & 5.6 & 5.0 \\
\hline \multirow[t]{2}{*}{ Mean } & & 4.3 & 4.2 & 4.4 & 4.8 & \\
\hline & & \multicolumn{5}{|c|}{$\mathbf{T} \times \mathbf{D}$} \\
\hline \multirow{2}{*}{\multicolumn{2}{|c|}{$\begin{array}{l}\text { SEd } \\
\text { CD }(P=0.05)\end{array}$}} & & 0.08 & 0.07 & \multicolumn{2}{|l|}{0.16} \\
\hline & & & $0.16 * *$ & $0.15 * *$ & \multicolumn{2}{|c|}{$\mathbf{0 . 3 3} * *$} \\
\hline
\end{tabular}

Table.2 Standardization of seed biopriming using Azotobacter for snakegourd cv. $\mathrm{CO}_{2^{-}}$ Germination

\begin{tabular}{|c|c|c|c|c|c|c|}
\hline \multirow{3}{*}{\multicolumn{2}{|c|}{$\begin{array}{l}\text { Biopriming treatments } \\
\text { (T) }\end{array}$}} & \multicolumn{5}{|c|}{ Germination (\%) } \\
\hline & & \multicolumn{5}{|c|}{ Soaking duration in $h(D)$} \\
\hline & & 12 & 18 & 24 & 30 & Mean \\
\hline \multicolumn{2}{|c|}{ Nonprimed seed } & $\begin{array}{c}61 \\
(51.35)\end{array}$ & $\begin{array}{c}61 \\
(51.35)\end{array}$ & $\begin{array}{c}61 \\
(51.35)\end{array}$ & $\begin{array}{c}61 \\
(51.35)\end{array}$ & $\begin{array}{c}61 \\
(51.35)\end{array}$ \\
\hline \multicolumn{2}{|c|}{ Hydropriming } & $\begin{array}{c}71 \\
(57.41)\end{array}$ & $\begin{array}{c}75 \\
(60.50)\end{array}$ & $\begin{array}{c}66 \\
(54.33)\end{array}$ & $\begin{array}{c}61 \\
(51.65)\end{array}$ & $\begin{array}{c}68 \\
(55.97)\end{array}$ \\
\hline \multirow[t]{3}{*}{ Azotobacter } & $10 \%$ & $\begin{array}{c}63 \\
(52.53)\end{array}$ & $\begin{array}{c}67 \\
(55.24)\end{array}$ & $\begin{array}{c}72 \\
(58.05)\end{array}$ & $\begin{array}{c}74 \\
(59.83)\end{array}$ & $\begin{array}{c}69 \\
(56.41)\end{array}$ \\
\hline & $15 \%$ & $\begin{array}{c}64 \\
(53.13)\end{array}$ & $\begin{array}{c}68 \\
(55.55)\end{array}$ & $\begin{array}{c}73 \\
(58.69)\end{array}$ & $\begin{array}{c}76 \\
(60.68)\end{array}$ & $\begin{array}{c}70 \\
(57.01)\end{array}$ \\
\hline & $20 \%$ & $\begin{array}{c}65 \\
(53.88)\end{array}$ & $\begin{array}{c}70 \\
(56.79)\end{array}$ & $\begin{array}{c}75 \\
(60.00)\end{array}$ & $\begin{array}{c}80 \\
(63.27)\end{array}$ & $\begin{array}{c}73 \\
(58.48)\end{array}$ \\
\hline \multirow[t]{2}{*}{ Mean } & & $\begin{array}{c}65 \\
(53.66) \\
\end{array}$ & $\begin{array}{c}68 \\
(55.88) \\
\end{array}$ & $\begin{array}{c}69 \\
(56.48) \\
\end{array}$ & $\begin{array}{c}71 \\
(57.36) \\
\end{array}$ & \\
\hline & & & $\mathbf{T}$ & D & T x D & $\mathrm{F}$ \\
\hline \multicolumn{2}{|l|}{$\begin{array}{l}\text { SEd } \\
\text { CD }(P=0.05)\end{array}$} & & $\begin{array}{l}0.26 \\
0.52 * *\end{array}$ & $\begin{array}{l}0.23 \\
0.47 * *\end{array}$ & $\begin{array}{l}0.52 \\
1.05 * *\end{array}$ & \\
\hline
\end{tabular}

(Figures in parentheses indicate arcsine values) 
Table.3 Standardization of seed biopriming using Azotobacter for snakegourd cv. $\mathrm{CO}_{2}{ }^{-}$ Root length

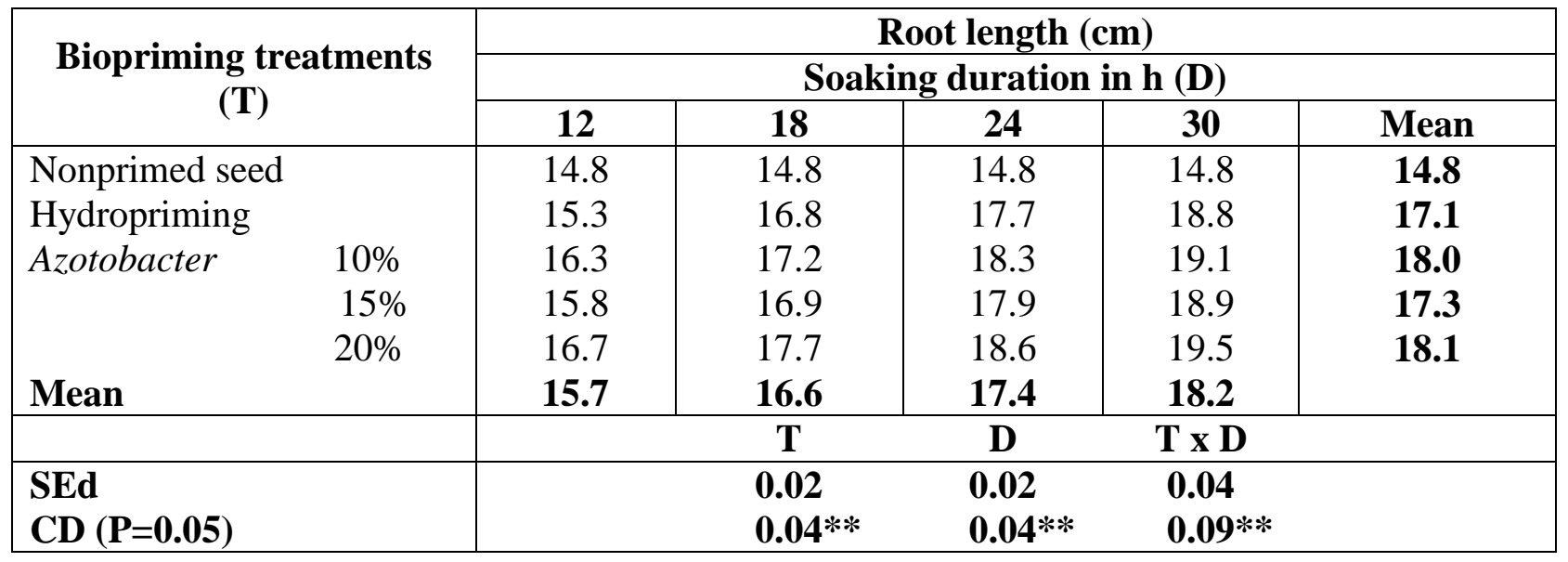

Table.4 Standardization of seed biopriming using Azotobacter for snakegourd cv. $\mathrm{CO}_{2}-$ Shoot length

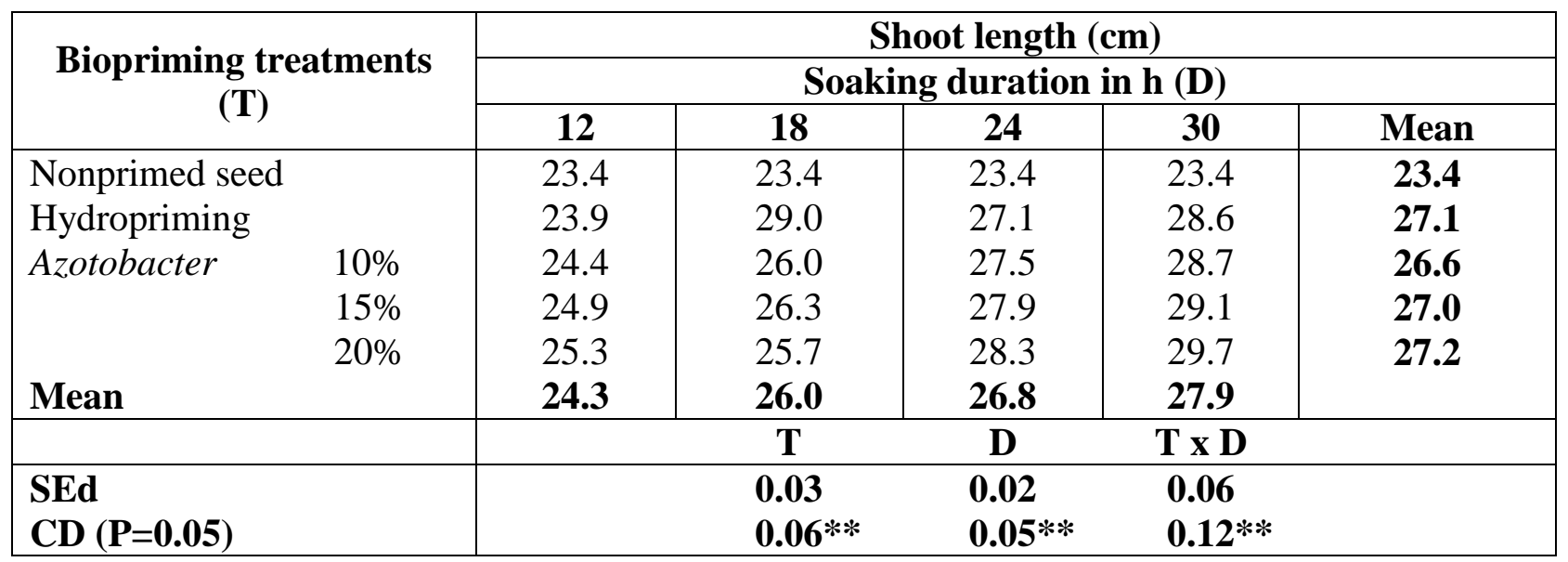

Table.5 Standardization of seed biopriming using Azotobacter for snakegourd cv. $\mathrm{CO}_{2}$ drymatter production

\begin{tabular}{|c|c|c|c|c|c|}
\hline \multirow{3}{*}{ Biopriming treatments $(T)$} & \multicolumn{5}{|c|}{ Drymatter production ( $\left(10\right.$ seedlings ${ }^{-1}$ ) } \\
\hline & \multicolumn{5}{|c|}{ Soaking duration in $\mathrm{h}(\mathrm{D})$} \\
\hline & 12 & 18 & 24 & 30 & Mean \\
\hline Nonprimed seed & 1.116 & 1.116 & 1.116 & 1.116 & 1.116 \\
\hline Hydropriming & 1.156 & 1.171 & 1.142 & 1.121 & 1.147 \\
\hline \multirow[t]{3}{*}{ Azotobacter } & 1.123 & 1.151 & 1.147 & 1.160 & 1.145 \\
\hline & 1.129 & 1.152 & 1.163 & 1.181 & 1.156 \\
\hline & 1.156 & 1.154 & 1.166 & 1.186 & 1.165 \\
\hline \multirow[t]{2}{*}{ Mean } & 1.136 & 1.148 & 1.146 & 1.152 & \\
\hline & \multicolumn{2}{|r|}{$\mathbf{T}$} & D & \multicolumn{2}{|l|}{ TXD } \\
\hline $\begin{array}{l}\text { SEd } \\
\text { CD }(P=0.05)\end{array}$ & & 0.001 & 0.001 & \multicolumn{2}{|l|}{0.002} \\
\hline
\end{tabular}


Table.6 Standardization of seed biopriming using Azotobacter for snakegourd cv. $\mathrm{CO}_{2}-$ Vigour index

\begin{tabular}{|c|c|c|c|c|c|c|}
\hline \multirow{3}{*}{\multicolumn{2}{|c|}{ Biopriming treatments $(\mathrm{T})$}} & \multicolumn{5}{|c|}{ Vigour index (Germination x Seedling length) } \\
\hline & & \multicolumn{5}{|c|}{ Soaking duration in $h(D)$} \\
\hline & & 12 & 18 & 24 & 30 & Mean \\
\hline Nonprimed & & 2334 & 2334 & 2334 & 2334 & 2334 \\
\hline Hydroprimir & & 2787 & 3469 & 2958 & 2915 & 3082 \\
\hline \multirow[t]{3}{*}{ Azotobacter } & $10 \%$ & 2570 & 2919 & 3298 & 3583 & 3168 \\
\hline & $15 \%$ & 2611 & 2941 & 3349 & 3655 & 3226 \\
\hline & $20 \%$ & 2747 & 3043 & 3474 & 3847 & 3390 \\
\hline \multirow{2}{*}{\multicolumn{2}{|c|}{ Mean }} & 2610 & 2941 & 3083 & 3267 & \\
\hline & & \multicolumn{2}{|r|}{$\mathbf{T}$} & D & \multicolumn{2}{|l|}{ TXD } \\
\hline \multicolumn{2}{|l|}{ SEd } & & 11.6 & 10.4 & \multicolumn{2}{|l|}{23.3} \\
\hline
\end{tabular}

Table.7 Standardization of seed biopriming using Azospirillum for snakegourd cv. $\mathrm{CO}_{2}-$ Speed of germination

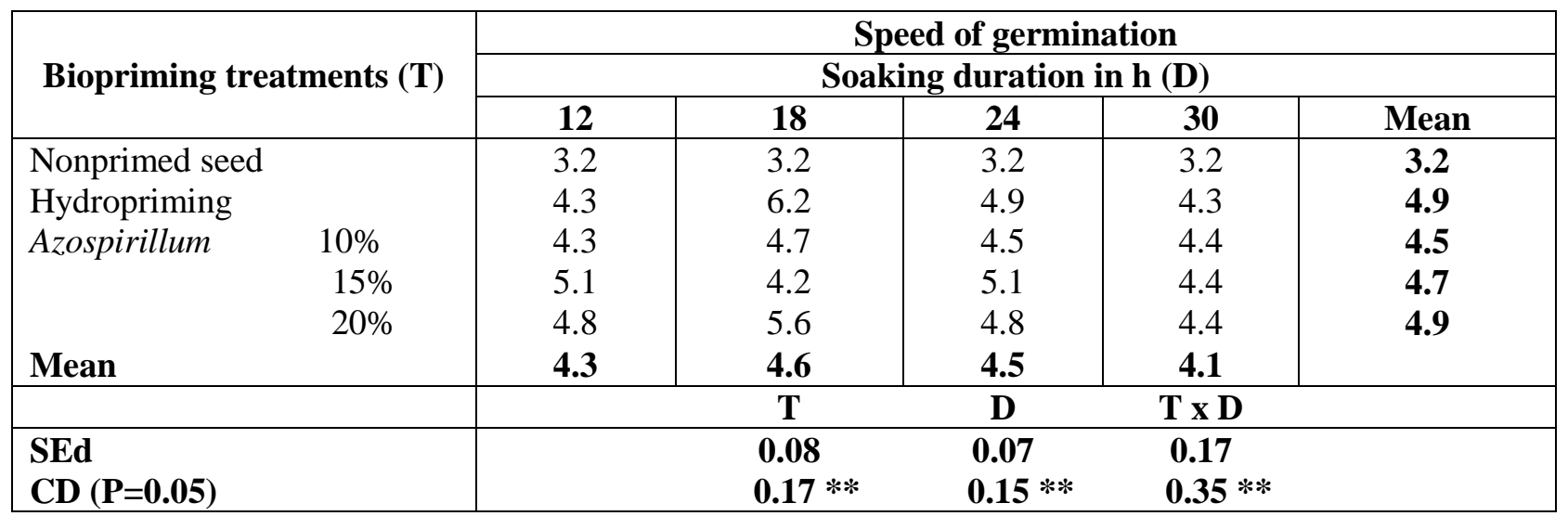

Table.8 Standardization of seed biopriming using Azospirillum for snakegourd cv. $\mathrm{CO}_{2}-$ Germination

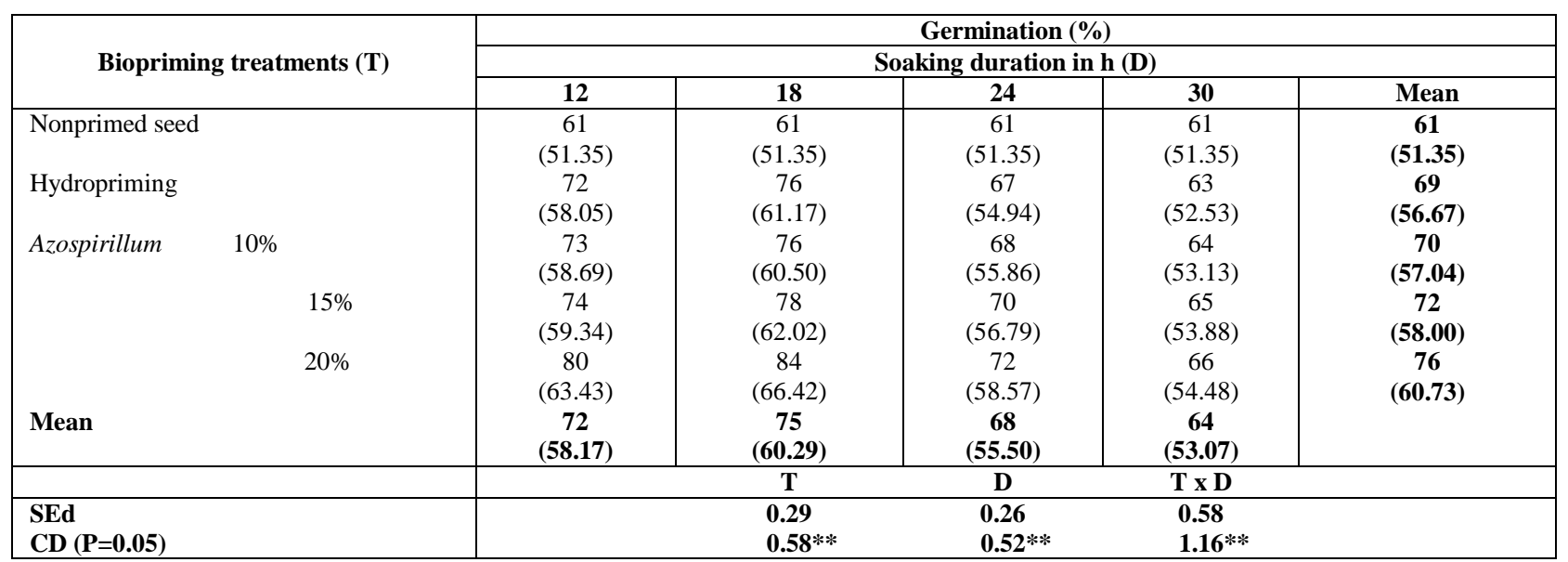

(Figures in parentheses indicate arcsine values) 
Table.9 Standardization of seed biopriming using Azospirillum for snakegourd cv. $\mathrm{CO}_{2}-$ Root length

\begin{tabular}{|c|c|c|c|c|c|c|}
\hline \multirow{3}{*}{\multicolumn{2}{|c|}{$\begin{array}{l}\text { Biopriming treatments } \\
\text { (T) }\end{array}$}} & \multicolumn{5}{|c|}{ Root length $(\mathrm{cm})$} \\
\hline & & \multicolumn{5}{|c|}{ Soaking duration in $\mathrm{h}(\mathrm{D})$} \\
\hline & & 12 & 18 & 24 & 30 & Mean \\
\hline Nonprimed s & & 14.9 & 14.9 & 14.9 & 14.9 & 14.9 \\
\hline Hydroprimin & & 18.0 & 19.0 & 16.8 & 15.3 & 17.2 \\
\hline \multirow[t]{3}{*}{ Azospirillum } & $10 \%$ & 18.5 & 19.6 & 17.5 & 16.5 & 18.0 \\
\hline & $15 \%$ & 18.1 & 19.3 & 17.1 & 15.8 & 17.5 \\
\hline & $20 \%$ & 18.7 & 20.0 & 17.8 & 16.8 & 18.3 \\
\hline \multirow[t]{2}{*}{ Mean } & & $\mathbf{1 7 . 6}$ & 18.5 & 16.8 & 15.8 & \\
\hline & & \multicolumn{2}{|r|}{$\mathbf{T}$} & D & \multicolumn{2}{|l|}{ T x D } \\
\hline \multirow{2}{*}{\multicolumn{2}{|c|}{$\begin{array}{l}\text { SEd } \\
\text { CD }(P=0.05)\end{array}$}} & & $\mathbf{0 . 0 3}$ & 0.02 & \multirow{2}{*}{\multicolumn{2}{|c|}{$\begin{array}{l}0.06 \\
0.12 * *\end{array}$}} \\
\hline & & & $0.06 * *$ & $0.05 * *$ & & \\
\hline
\end{tabular}

Table.10 Standardization of seed biopriming using Azospirillum for snakegourd cv. $\mathrm{CO}_{2}-$ Shoot length

\begin{tabular}{|c|c|c|c|c|c|c|}
\hline \multirow{3}{*}{\multicolumn{2}{|c|}{$\begin{array}{l}\text { Biopriming treatments } \\
\text { (T) }\end{array}$}} & \multicolumn{5}{|c|}{ Shoot length $(\mathrm{cm})$} \\
\hline & & \multicolumn{5}{|c|}{ Soaking duration in $h(D)$} \\
\hline & & 12 & 18 & 24 & 30 & Mean \\
\hline Nonprimed s & & 23.8 & 23.8 & 23.8 & 23.8 & 23.8 \\
\hline Hydroprimin & & 27.4 & 29.0 & 26.0 & 24.2 & 26.6 \\
\hline Azospirillum & $10 \%$ & 27.1 & 29.7 & 25.6 & 24.7 & 26.7 \\
\hline & $15 \%$ & 27.8 & 29.2 & 26.3 & 25.0 & 27.0 \\
\hline & $20 \%$ & 28.5 & 30.2 & 26.0 & 24.2 & 27.2 \\
\hline \multirow[t]{2}{*}{ Mean } & & 26.9 & 28.3 & 25.5 & 24.4 & \\
\hline & & \multicolumn{2}{|r|}{$\mathbf{T}$} & D & \multicolumn{2}{|l|}{ T x D } \\
\hline \multirow{2}{*}{\multicolumn{2}{|c|}{$\begin{array}{l}\text { SEd } \\
\text { CD }(P=0.05)\end{array}$}} & & 0.04 & 0.04 & \multirow{2}{*}{\multicolumn{2}{|c|}{$\begin{array}{l}0.09 \\
0.18 * *\end{array}$}} \\
\hline & & & $0.09 * *$ & $0.08 * *$ & & \\
\hline
\end{tabular}

Table.11 Standardization of seed biopriming using Azospirillum for snakegourd cv. $\mathrm{CO}_{2}$ drymatter production

\begin{tabular}{|c|c|c|c|c|c|c|}
\hline \multirow{3}{*}{\multicolumn{2}{|c|}{ Biopriming treatments $(\mathrm{T})$}} & \multicolumn{5}{|c|}{ Drymatter production (g 10 seedlings $^{-1}$ ) } \\
\hline & & \multicolumn{5}{|c|}{ Soaking duration in $h(D)$} \\
\hline & & 12 & 18 & 24 & 30 & Mean \\
\hline Nonprimed s & & 1.117 & 1.117 & 1.117 & 1.117 & 1.117 \\
\hline Hydroprimin & & 1.157 & 1.172 & 1.143 & 1.122 & 1.148 \\
\hline \multirow[t]{3}{*}{ Azospirillum } & $10 \%$ & 1.161 & 1.152 & 1.148 & 1.124 & 1.146 \\
\hline & $15 \%$ & 1.164 & 1.182 & 1.153 & 1.130 & 1.157 \\
\hline & $20 \%$ & 1.167 & 1.187 & 1.155 & 1.135 & 1.161 \\
\hline \multirow[t]{2}{*}{ Mean } & & 1.153 & 1.162 & 1.143 & 1.125 & \\
\hline & & \multicolumn{2}{|r|}{$\mathbf{T}$} & D & \multicolumn{2}{|l|}{ TXD } \\
\hline \multicolumn{2}{|l|}{$\begin{array}{l}\text { SEd } \\
\text { CD }(P=0.05)\end{array}$} & & $\begin{array}{l}\mathbf{0 . 0 0 1} \\
\mathbf{0 . 0 0 2} * *\end{array}$ & $\begin{array}{l}0.001 \\
0.002 * *\end{array}$ & \multicolumn{2}{|l|}{$\begin{array}{l}0.002 \\
0.004 * *\end{array}$} \\
\hline
\end{tabular}


Table.12 Standardization of seed biopriming using Azospirillum for snake gourd cv. $\mathrm{CO}_{2}$ Vigour index

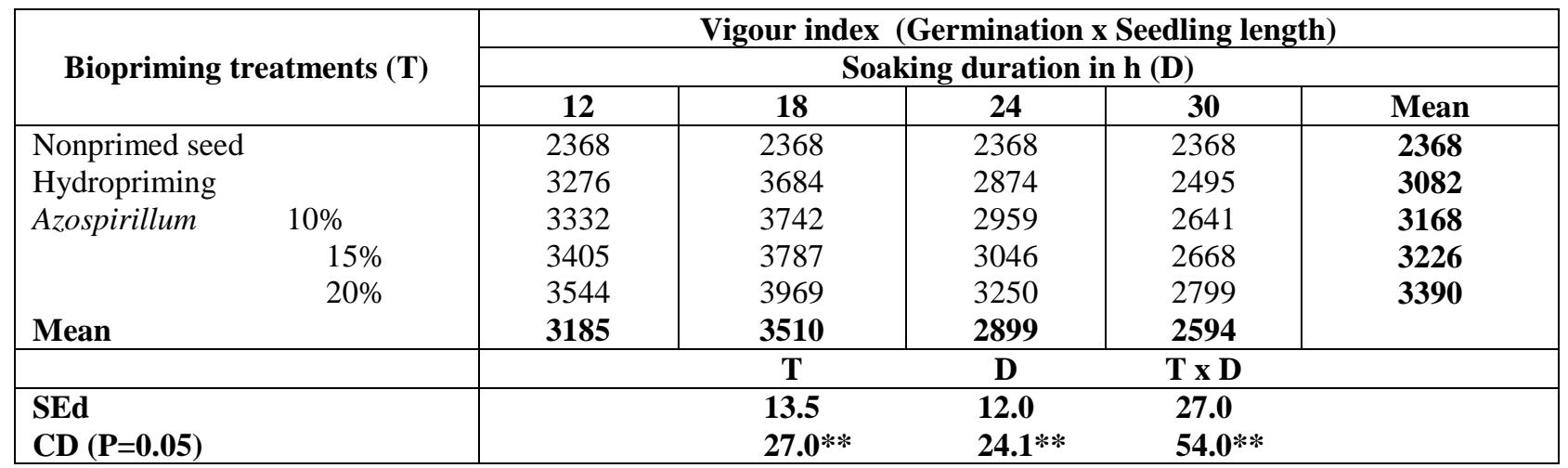

Table.13 Standardization of seed biopriming using phosphobacteria for snakegourd cv. $\mathrm{CO}_{2}-$ Speed of germination

\begin{tabular}{|c|c|c|c|c|c|}
\hline \multirow{3}{*}{ Biopriming treatments $(T)$} & \multicolumn{5}{|c|}{ Speed of germination } \\
\hline & \multicolumn{5}{|c|}{ Soaking duration in $\mathrm{h}$ (D) } \\
\hline & 12 & 18 & 24 & 30 & Mean \\
\hline Nonprimed seed & 3.2 & 3.2 & 3.2 & 3.2 & 3.2 \\
\hline Hydropriming & 4.3 & 4.2 & 4.9 & 4.3 & 4.4 \\
\hline phosphobacteria & 4.3 & 4.6 & 4.4 & 4.3 & 4.4 \\
\hline $15 \%$ & 4.8 & 5.6 & 4.7 & 4.4 & 4.9 \\
\hline $20 \%$ & 5.0 & 5.5 & 4.6 & 4.5 & 4.9 \\
\hline \multirow[t]{2}{*}{ Mean } & 4.3 & 4.6 & 4.3 & 4.1 & \\
\hline & & $\mathbf{T}$ & D & TXD & \\
\hline $\begin{array}{l}\text { SEd } \\
\text { CD }(P=0.05)\end{array}$ & & $\begin{array}{l}\mathbf{0 . 0 8} \\
\mathbf{0 . 1 6} * *\end{array}$ & $\begin{array}{l}0.07 \\
0.15 * *\end{array}$ & $\begin{array}{l}0.16 \\
0.33 * *\end{array}$ & \\
\hline
\end{tabular}

Table.14 Standardization of seed biopriming using phosphobacteria for snakegourd cv. $\mathrm{CO}_{2}$ - Germination

\begin{tabular}{|c|c|c|c|c|c|}
\hline \multirow{3}{*}{ Biopriming treatments $(T)$} & & & minatio & & \\
\hline & \multicolumn{5}{|c|}{ Soaking duration in $h(D)$} \\
\hline & 12 & 18 & 24 & 30 & Mean \\
\hline Nonprimed seed & 61 & 61 & 61 & 61 & 61 \\
\hline \multirow{2}{*}{ Hydropriming } & 71 & 75 & 66 & 61 & 68 \\
\hline & $(57.41)$ & $(60.50)$ & (54.33) & $(51.65)$ & $(\mathbf{5 5 . 9 7})$ \\
\hline phosphobacteria $10 \%$ & $\begin{array}{c}64 \\
(5313)\end{array}$ & 68 & $\begin{array}{c}73 \\
(5869)\end{array}$ & $\begin{array}{c}76 \\
(6068)\end{array}$ & 70 \\
\hline \multirow[t]{2}{*}{$15 \%$} & $\begin{array}{c}65 \\
(53.88)\end{array}$ & $\begin{array}{c}78 \\
(62.02)\end{array}$ & $\begin{array}{c}74 \\
(59.34)\end{array}$ & $\begin{array}{c}70.00) \\
(56.79)\end{array}$ & $\begin{array}{c}72 \\
(58.00)\end{array}$ \\
\hline & $\begin{array}{c}63 \\
(5253)\end{array}$ & 67 & $\begin{array}{c}72 \\
(5805)\end{array}$ & $\begin{array}{c}74 \\
(5983)\end{array}$ & 69 \\
\hline \multirow[t]{2}{*}{ Mean } & $\begin{array}{c}65 \\
(53.66)\end{array}$ & $\begin{array}{c}70 \\
(56.93)\end{array}$ & $\begin{array}{c}69 \\
(56.35)\end{array}$ & $\begin{array}{c}68 \\
(56.06)\end{array}$ & \\
\hline & & $\mathbf{T}$ & D & T X D & \\
\hline \multirow{2}{*}{$\begin{array}{l}\text { SEd } \\
\text { CD }(P=0.05)\end{array}$} & & 0.24 & 0.21 & 0.48 & \\
\hline & & $0.48 * *$ & $\mathbf{0 . 4 3}$ ** & $0.97 *:$ & \\
\hline
\end{tabular}

(Figures in parentheses indicate arcsine values) 
Table.15 Standardization of seed biopriming using phosphobacteria for snakegourd cv. $\mathrm{CO}_{2}$ - Root length

\begin{tabular}{|c|c|c|c|c|c|c|}
\hline \multirow{3}{*}{\multicolumn{2}{|c|}{$\begin{array}{l}\text { Biopriming treatments } \\
\text { (T) }\end{array}$}} & \multicolumn{5}{|c|}{ Root length $(\mathrm{cm})$} \\
\hline & & \multicolumn{5}{|c|}{ Soaking duration in $h$ (D) } \\
\hline & & 12 & 18 & 24 & 30 & Mean \\
\hline Nonprimed seed & & 12.7 & 12.7 & 12.7 & 12.7 & 12.7 \\
\hline Hydropriming & & 13.3 & 18.1 & 17.4 & 15.4 & 16.0 \\
\hline phosphobacteria & $10 \%$ & 13.8 & 18.5 & 17.1 & 15.8 & 16.3 \\
\hline & $15 \%$ & 14.6 & 19.8 & 17.6 & 16.5 & 17.1 \\
\hline & $20 \%$ & 14.2 & 18.8 & 17.6 & 16.2 & 16.7 \\
\hline \multirow{2}{*}{\multicolumn{2}{|c|}{ Mean }} & 13.7 & $\mathbf{1 7 . 5}$ & 16.4 & 15.3 & \\
\hline & & \multicolumn{5}{|c|}{ T x D } \\
\hline \multicolumn{2}{|l|}{$\begin{array}{l}\text { SEd } \\
\text { CD }(P=0.05)\end{array}$} & & $\begin{array}{l}0.02 \\
0.05 * *\end{array}$ & $\begin{array}{l}\mathbf{0 . 0 2} \\
\mathbf{0 . 0 4} * *\end{array}$ & \multicolumn{2}{|l|}{$\begin{array}{l}0.05 \\
0.10 * *\end{array}$} \\
\hline
\end{tabular}

Table.16 Standardization of seed biopriming using phosphobacteria for snakegourd cv. $\mathrm{CO}_{2}$ - Shoot length

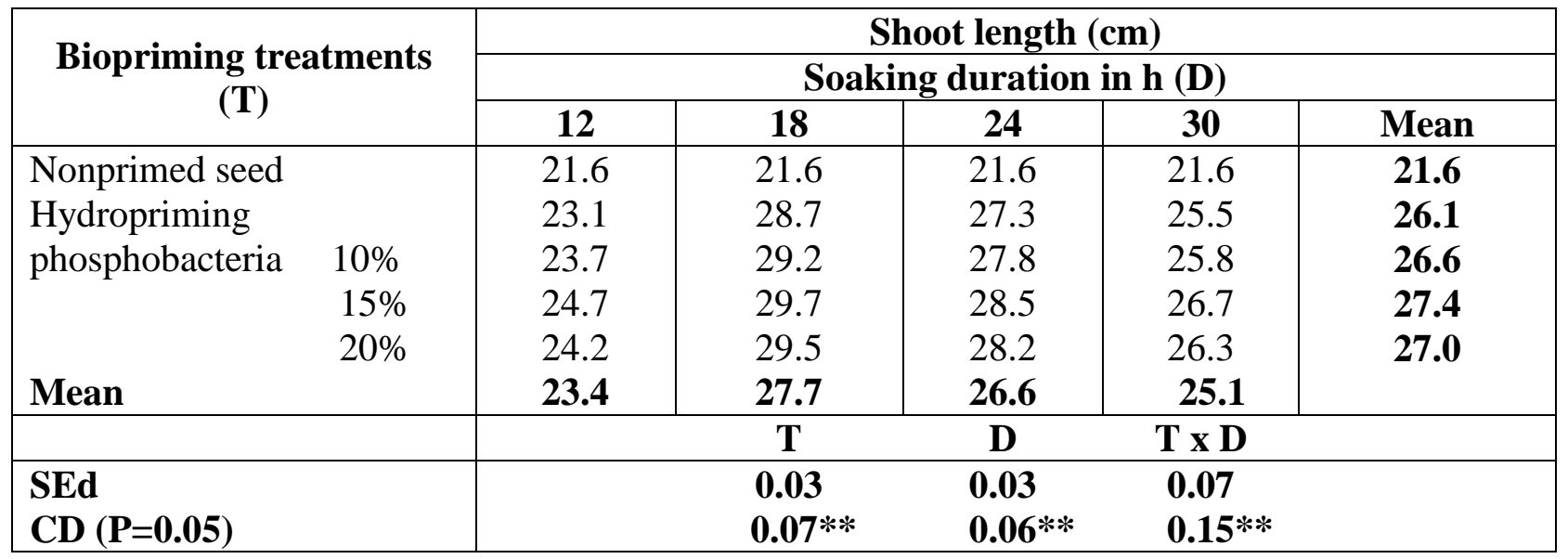

Table.17 Standardization of seed biopriming using phosphobacteria for snakegourd $\mathrm{CO}_{2}$ - Drymatter production

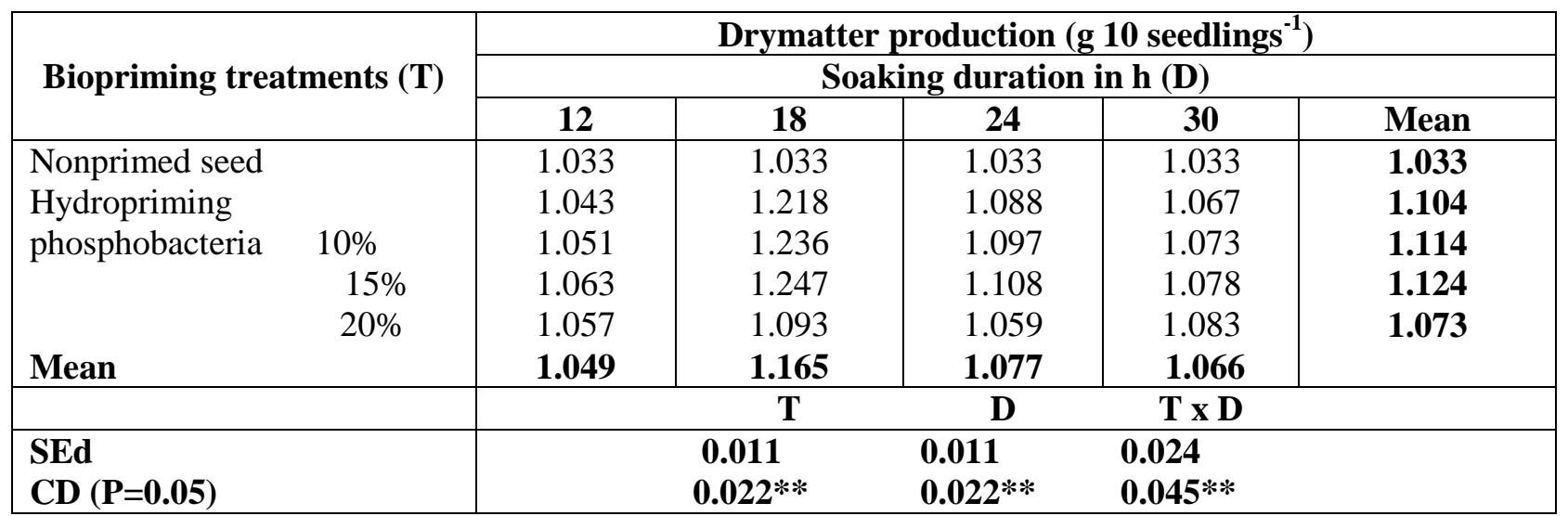


Table.18 Standardization of seed biopriming using phosphobacteria for Snakegourd cv. $\mathrm{CO}_{2}$ - Vigour index

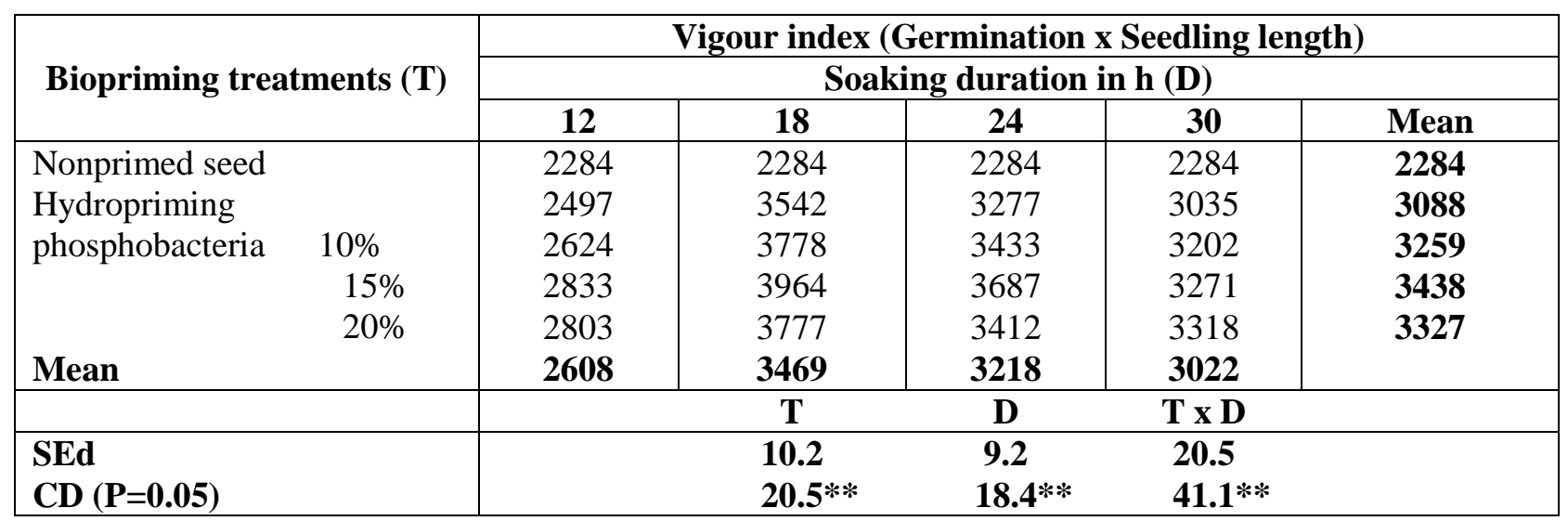

Ramamoorthy et al., (2000) reported that seed biofortification with Azospirillum enhanced seedling vigour encompassing speed of germination, seedling length and dry weight of high and low vigour seed lots in rice.

Thamizh Vendan and Thangaraju (2007) inoculated the tomato seeds with liquid formulation of Azospirillum which enhanced the plant height, biomass, nitrogen uptake of plants, available nitrogen content of soil and yield of tomato.

Kavitha (2011) reported that rice ADT 43 seeds bioprimed with liquid Azospirillum 20\% for $12 \mathrm{~h}$ expressed high values for speed of germination and vigour based on dry matter production accounted for 32 and 7 per cent increase over nonprimed seed. However, the seeds bioprimed with Azospirillum 20\% for 6h showed increased germination and vigour based on seedling growth accounted for 11 and 18 per cent increase over nonprimed seed.

Significant variations were observed in Speed of germination, Germination, Drymatter production, seedling length and vigour index by biopriming treatments, durations of biopriming and their interactions. The interactions between the biopriming treatments and durations of biopriming expressed that the seeds bioprimed using phosphobacteria $15 \%$ for $18 \mathrm{~h}$ registered high speed of germination (5.6) followed by phosphobacteria $20 \%$ for $18 \mathrm{~h}(5.5)$. The speed of germination of nonprimed seeds was 3.2. The interactions effects indicated that seeds bioprimed with phosphobacteria at $15 \%$ for $18 \mathrm{~h}$ registered higher germination of 78 per cent as compared to nonprimed seeds which was 61 per cent.

Seeds bioprimed with phosphobacteria $15 \%$ for $18 \mathrm{~h}$ measured longer root $(19.8 \mathrm{~cm})$ than nonprimed seeds $(12.7 \mathrm{~cm})$. The interactions between biopriming treatments and durations of biopriming showed that phosphobacteria $20 \%$ for $18 \mathrm{~h}$ recorded the longest shoot of $29.7 \mathrm{~cm}$. The shortest shoot of $21.6 \mathrm{~cm}$ was in nonprimed seeds.

The interactions between biopriming treatments and durations of biopriming expressed that the biopriming involving phosphobacteria $15 \%$ for $18 \mathrm{~h}$ registered more drymatter production $(1.247 \mathrm{~g} 10$ seedlings ${ }^{-1}$ ).

The less drymatter production of nonprimed seeds was $1.033 \mathrm{~g} 10$ seedlings $^{-1}$. Phosphobacteria biopriming at $15 \%$ for $12 \mathrm{~h}$ registered more vigour (3964) when compared to nonprimed seeds (2284).

Gomathy et al., (2009) reported that $1 \%$ inoculum showed better results followed by $1.5 \%$ inoculum for the optimization of liquid phosphobacteria required for maize seeds. 
Mariselvam (2012) reported that bhendi seed bioprimed with liquid phosphobacteria $20 \%$ for $12 \mathrm{~h}$ was found to be the best seed treatment to improve the seed quality. Seeds bioprimed with Azotobacter $20 \%$ for $30 \mathrm{~h}$ was the best and suitable biopriming treatment. This treatment showed a higher per centage of increase over the nonprimed seeds and they were 93, 19, 32, 27, 6 and 65 per cent for speed of germination, germination, root length, shoot length, drymatter production and vigour index respectively.

Seeds bioprimed with liquid Azospirillum 20\% for $18 \mathrm{~h}$ expressed high values for speed of germination and vigour index which accounted for 75 and 68 per cent increase over nonprimed seed. The increase in germination, root length, shoot length and drymatter production accounted for 23, 34, 27 and 63 per cent over nonprimed seed.

Seeds bioprimed with phosphobacteria $15 \%$ for $18 \mathrm{~h}$ was found to improve the speed of germination, germination, root length, shoot length, drymatter production and vigour index. The increases over nonprimed seeds for these parameters were $75,17,56,38,21$ and 74 per cent respectively.

Among the different treatments, concentrations and durations of Azotobacter $20 \%$ for $30 \mathrm{~h}$ or liquid Azospirillum $20 \%$ for $18 \mathrm{~h}$ or liquid phosphobacteria 15\%. Seed biopriming treatments with Azospirillum $20 \%$ for $18 \mathrm{~h}$ was the best and suitable biopriming treatment to enhance the germination rate, total germination percentage, seedling growth and vigour.

\section{References}

Abdul-Baki, A.A. and J.D. Anderson. 1973. Vigour deterioration of soybean seeds by multiple criteria. Crop Sci., 13: 630-633.

Gomathy, M., M. Thangaraju and C. Sarathambal. 2009. Optimization of liquid phosphobacteria required for maize seeds. Asian Journal of Bio. Sci., 4(1): 53-55.

ISTA, 2009. International Rules for Seed Testing. International Seed Testing Association, Bassersdorf, Switzerland.

Kavitha, S. 2011. Biopriming with biocontrol agents and liquid biofertilizers for rice seed cv. ADT 43. M.Sc. (Ag.) Thesis, Tamil Nadu Agricultural University, Coimbatore.

Maguire, J.D. 1962. Speed of germination - Aid in selection and evaluation of seedling emergence and vigour. Crop Sci., 2: 176177.

Mariselvam, D. 2012. Biopriming with biocontrol agents and liquid biofertilizers for bhendi seed cv. ADT 43. M.Sc. (Ag.) Thesis, Tamil Nadu Agricultural University, Coimbatore.

Panse, V.G. and P.V. Sukatme. 1985. Statistical methods for agricultural workers. ICAR publication, New Delhi, 359.

Natarajan K.N., and A. Lakshmanan. 2000. Seed biofortification with Azospirillum spp. for improvement of seedling vigour and productivity in rice (Oryza sativa L.). Seed Sci. Technol., 28: 809-815.

Ramamoorthy, K., N. Natarajan and A. Lakshmanan. 2000. Seed biofortification with Azospirillum spp. for improvement of seedling vigour and productivity in rice (Oryza sativa L.). Seed Sci. Technol., 28: 809-815.

Thamizh Vendan, R.T. and M. Thangaraju. 2007. Influence of liquid and cyst formulations of Azospirillum with inorganic fertilizers on the growth and yield of tomato. Afr. J. Biotech., 6(19): 30-35.

\section{How to cite this article:}

Gowthamy, U., P. Selvaraju and Hemalatha, G. 2017. Standardization of Seed Biopriming with Liquid Biofertilizers on Snake Gourd (Trichosanthes cucumerina). Int.J.Curr.Microbiol.App.Sci. 6(12): 2513-2524. doi: https://doi.org/10.20546/ijcmas.2017.612.292 